\title{
Ablação por Cateter do Flutter Atrial. Caracterização Eletrofisiológica da Interrupção da Condução pelos Istmos Posterior e Septal
}

\author{
José Marcos Moreira, Sílvio Roberto Borges Alessi, André Gustavo da Silva Rezende, \\ Luis Alberto Renjel Prudêncio, Angelo Amato Vincenzo de Paola
}

São Paulo, SP

\begin{abstract}
Objetivo - Avaliar os tipos de bloqueio obtidos nos istmos posterior (entre o anel tricuspídeo e veia cava inferior) e septal (entre o anel tricuspídeo e óstio do seio coronário), após ablação do flutter atrial (FLA).

Métodos - Foram submetidos à ablação por radiofreqüência (RF) 14 pacientes com FLA tipo I (9 homens) em 16 procedimentos. A ativação atrial ao redor do anel tricuspideo foi avaliada em ritmo sinusal utilizando-se cateter "Halo" com 10 pares de eletrodos (H12 a H19-20), durante estimulação do seio coronário proximal (SCP) e região póstero-lateral do átrio direito (H1-2), antes e após ablações lineares. De acordo com a frente de programação do impulso definiu-se: ausência de bloqueio (condução bidirecional), bloqueio incompleto (condução bidirecional com retardo num dos sentidos) e bloqueio completo (ausência de condução pelo istmo). O intervalo desta ativação $(\triangle S C P / H 1-2)$ foi analisado.

Resultados - Bloqueio completo foi obtido em 7 procedimentos (44\%) e incompleto em 4 (25\%). O $\triangle \mathrm{SCP} / \mathrm{H1}$ 2 foi de $74 \pm 26 m s$ no primeiro grupo e de 30,5 $\pm 7,5 \mathrm{~ms}$ no segundo $(p<0,05)$. Em 5 casos não demonstrou-se bloqueio. Num seguimento médio de 12 meses, ocorreu recorrência do FLA nos 6 pacientes com bloqueio incompleto ou ausente e em nenhum com bloqueio completo $(p<0,001)$.

Conclusão - A verificação de bloqueio completo pelo mapeamento multipolar atrial é uma estratégia eficaz para definir o sucesso e recorrência clínica na ablação do FLA tipo I.
\end{abstract}

Palavras-chave: flutter atrial, ablação por cateter, radiofrequiência

\section{Catheter Ablation of Atrial Flutter. Electrophysiological Characterization of Posterior and Septal Isthmus Block}

Purpose - Evaluate the different types of conduction blocks obtained between inferior vena cava-tricuspid annulus (posterior isthmus) and between tricuspid annulus-coronary sinus ostium (septal isthmus) after radiofrequency $(R F)$ catheter ablation of atrial flutter ( $A F L$ )

Methods - In 16 procedures, 14 patients (pts), 9 male, with type I AFL underwent RF ablation. Atrial activation around tricuspid annulus was performed with a 10-bipole "Halo" catheter (H1-2; H19-20). In sinus rhythm, isthmus conduction was evaluated during proximal coronary sinus (PCS) and low lateral right atrium (H1-2) pacing, before and after linear ablation. According to the wave front of impulse propagation we assessed absence of block (bidirectional conduction); incomplete block (bidirectional conduction with delay in one front of impulse propagation) and complete block (absence of conduction). The PCS/H12 interval was measured before and after ablation.

Results - Complete isthmus block was achieved in 7 (44\%) and incomplete block in 4 (25\%) procedures. Conduction block was not achieved in 5 procedures. At a mean follow-up of 12 months, there were no recurrences in the pts with complete block, whereas AFL recurred in the 6 pts with incomplete or no conduction block $(p<0.001)$. Pts with complete block had $\triangle$ PCS/H1-2 interval (74.0 \pm $26.0 \mathrm{~ms})$ greater than incomplete $(30.5 \pm 7.5 \mathrm{~ms})$ or absent block $(p<0.05)$.

Conclusion - The verification of complete isthmus conduction block with atrial multipolar mapping is an effective strategy to assess electrophysiological success and absence of late recurrence in common atrial flutter ablation.

Key-words: atrial flutter, catheter ablation, radiofrequency.

Arq Bras Cardiol, volume $71\left(\mathrm{n}^{0} 1\right), 37-47,1998$

Escola Paulista de Medicina - UNIFESP

Correspondência: José Marcos Moreira - Rua Napoleão de Barros, 593 - 04024-002

- São Paulo, SP

Recebido para publicação em 20/2/98

Aceito em 15/4/98
O flutter atrial (FLA) foi descrito no início do século como uma arritmia resultante de um circuito macroreentrante localizado no átrio direito (AD), ao redor de obstáculos naturais ${ }^{1,2}$. Essas observações foram, posteriormente, confirmadas por mapeamento multipolar, tanto em 
modelos animais, quanto em mapeamento intra-operatório em humanos ${ }^{3-6}$.

Estudos recentes têm demonstrado que esse circuito reentrante percorre os espaços compreendidos entre o anel tricuspídeo e a veia cava inferior (istmo posterior) ${ }^{7,8}$ e entre o anel tricuspídeo e o óstio do seio coronário (istmo septal) ${ }^{9}$. Esses espaços são preferenciais para a interrupção do circuito através de lesão induzida por radiofreqüência.

As séries iniciais de ablação de FLA utilizavam eletrogramas fracionados (zonas de condução lenta), bem como a presença de encarrilhamento oculto (localização de reentrada), como marcadores de sítios ótimos para aplicação de radiofreqüência ${ }^{3,10,11}$. Os critérios de sucesso eram a simples interrupção do FLA ou a inabilidade de reindução através de técnicas de estimulação atrial programada (EAP). No entanto as taxas de recorrência variavam entre 7\% a 44\% a despeito do sucesso inicial ${ }^{12,13}$.

Posteriormente, a realização de lesões lineares por cateter, utilizando a abordagem anatômica dos istmos posterior ou septal mostrou-se mais eficiente que as técnicas descritas $^{7,9}$. Novos marcadores eletrofisiológicos de sucesso foram criados, a partir de estudos utilizando mapeamento da ativação atrial ao redor do anel tricuspídeo, em ritmo sinusal, através de um cateter especial duodecapolar, demonstrando ser a presença de bloqueio completo ao nível do istmo um preditor de sucesso tardio mais eficaz ${ }^{14,15}$.

O objetivo do presente estudo foi avaliar os critérios de bloqueio nos istmos posterior e septal, durante e após a ablação do FLA, e correlacioná-los com a evolução clínica ao longo de um período de acompanhamento de 3 a 17 meses.

\section{Métodos}

Foram estudados 14 pacientes com FLA tipo I, 9 homens e 5 mulheres, com idades variando de 14 a 67 (média de 46) anos, submetidos à ablação por cateter no período de outubro/96 a outubro/97, totalizando 16 procedimentos.

Todos os pacientes tinham história prévia de palpitações taquicárdicas, com documentação eletrocardiográfica do FLA tipo I, com diagnóstico de FLA comum confirmado pela presença de ondas $f$ bifásicas e negativas nas derivações inferiores (DII, DIII e aVF), com frequiência variando de 240 a 340 bpm ${ }^{16}$. Todos foram submetidos a uma avaliação inicial que incluía história clínica, exame físico, ECG, radiografia de tórax, holter de $24 \mathrm{~h}$ e, na maioria dos casos, ecocardiograma bidimensional, com acompanhamento de 3 a 17 meses. A tabela I mostra as principais características dos pacientes.

Para o mapeamento, utilizou-se cateter 7 Fr deflectível, contendo 20 pares de eletrodos (H1-2 a H19-20), com distância intereletródica de $2 \mathrm{~mm}$ e distância entre os bipolos de $5 \mathrm{~mm}$ (cateter Halo, Cordis Webster) posicionado ao redor do anel tricuspídeo a fim de registrar a ativação atrial próxima às paredes póstero-lateral e posterior do AD. Um cateter quadripolar foi introduzido no seio coronário e um tripolar posicionado ao nível da região do feixe de His (fig. 1).

\begin{tabular}{|lcclc|}
\hline \multicolumn{5}{|c|}{ Tabela I -Características clínicas e ecocardiográficas dos } \\
pacientes \\
\hline Paciente & Idade & Sexo & Cardiopatia & FEVE (\%) \\
\hline 1 & 63 & F & Hipertensiva & 65 \\
2 & 43 & F & MCP chagásica & 46 \\
3 & 29 & F & DLM +PBM & 72 \\
4 & 14 & M & Ausente & - \\
5 & 35 & M & MCP dilatada & 59 \\
6 & 46 & M & Taquicardiomiopatias & 44 \\
7 & 39 & M & CIA corrigida & 67 \\
8 & 61 & M & Ausente & 67 \\
9 & 67 & M & Taquicardiomiopatias & 45 \\
10 & 64 & F & PVM & 35 \\
11 & 49 & F & MCP chagásica & 67 \\
12 & 59 & M & I M + PBM & - \\
13 & 46 & M & Ausente & - \\
14 & 34 & M & Ausente & 44 \\
\hline FEVE- fração de ejeção do ventrículo esquerdo; DLM- dupla lesão mitral; \\
PBM- prótese biológica mitral; MCP- miocardiopatia; CIA- comunica- \\
ção interatrial; PVM- prolapso de valva mitral; IM- insuficiência mitral. \\
\hline
\end{tabular}

Os eletrogramas foram filtrados entre 30 e $500 \mathrm{~Hz}$ e registrados em um polígrafo computadorizado (Lab 24 BARD Medical System, version 2.57), permitindo o registro e análise de até 18 canais simultaneamente, a 100 e $200 \mathrm{~mm} / \mathrm{s}$.

Odiagnóstico do tipo de FLA foi confirmado pela seqüência de ativação detectada pelo cateter duodecapolar (fig. 2).

Nos pacientes em ritmo sinusal (RS), o seio coronário proximal (SCP) foi estimulado a $600 \mathrm{~ms}$ de freqüência básica, com 2 vezes o limiar diastólico e $2 \mathrm{~ms}$ de largura de pulso, a fim de se verificar a sequiência de ativação do impulso nos sítios atriais, através da análise dos eletrogramas atriais registrados no cateter duodecapolar.

Em seguida, foi analisado o intervalo de tempo, em $\mathrm{ms,}$ percorrido pelo impulso gerado no SCP até a parede póstero-lateral do $\mathrm{AD}(\mathrm{H} 1-2)$, bem como o intervalo gerado pela estimulação da parede póstero-lateral até o SCP. A estimulação do SCP foi escolhida por apresentar boa estabilidade do cateter e baixo limiar de captura, permitindo boa visibilização da criação da linha de bloqueio, quando combinado com o mapeamento do anel tricuspídeo. Todas essas medidas foram realizadas antes e após a ablação.

Nos pacientes que iniciaram o exame em FLA, procedeu-se a aplicação de energia de RF, procurando se realizar linha de bloqueio, durante a arritmia. Se numa dessas aplicações houvesse quebra do FLA, procedíamos às medidas dos intervalos basais já citados. Caso contrário, o paciente era excluído do protocolo. Em nenhum paciente foi utilizado isoproterenol para avaliar a condução no istmo pós ablação.

O procedimento de ablação por cateter do FLA foi realizado conforme técnica previamente descrita ${ }^{9,15}$, utilizando-se preferencialmente as projeções oblíquas esquerda e direita $30^{\circ}$. Os cateteres de ablação utilizados foram o de ponta eletródica de $8 \mathrm{~mm}$ (Blazer, EP Technology) ou de $4 \mathrm{~mm}$ (Cordis, Webster). Os geradores de radiofreqüência utilizados foram os modelos Radionics RFG - 3C e EPT BOOT. 
As aplicações de radiofreqüência foram feitas com controle de potência e temperatura, com 20 a 30 w durante 40 a 60s. Durante a aplicação de radiofrequiência, o cateter de ablação (CA) foi progressivamente deslocado, partindo-se do anel tricuspídeo (registro de eletrogramas atrial e ventricular, com predomínio do último), durante fluoroscopia e mapeamento intracavitário, realizando-se paradas seqüenciais, até a junção do $\mathrm{AD}$ com a veia cava inferior (onde não mais se registrava atividade atrial no eletrodo do CA). No caso de insucesso por esta abordagem, a ablação era direcionada ao istmo compreendido entre $o$ anel tricuspídeo e o óstio do seio coronário.

Definições - A condução do estímulo antes e após a ablação do FLA pode ser definida estrategicamente utilizando-se medidas eletrofisiológicas obtidas através de estimulação do SCP e parede póstero-lateral do AD.

Durante estimulação do SCP $(600 \mathrm{~ms})$, foram considerados: a) ausência de bloqueio (fig. 3A) quando estimulado SCP contatavam-se duas frentes de ativação registradas no cateter duodecapolar, sendo que a $1^{\mathrm{a}}$ onda de ativação ascende a parede septal do $\mathrm{AD}$ em sentido anti-horário e a $2^{\mathrm{a}}$ onda de ativação propaga-se em direção ao istmo posterior, em sentido horário. A colisão das frentes de ativação ocorria ao nível de $\mathrm{AD}$ alto (H9-10); b) bloqueio incompleto
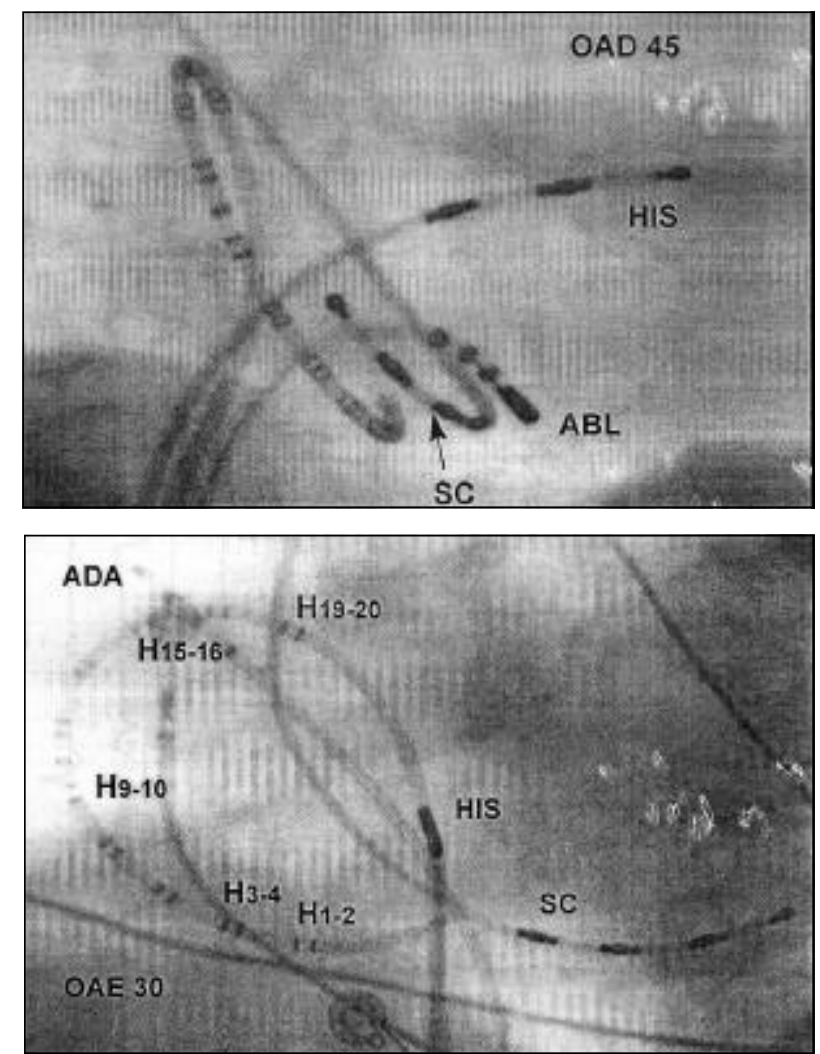

Fig. 1 - Posicionamento do cateter duodecapolar (Halo) no anel tricuspídeo em (A) $\mathrm{OAD} 45^{\circ}$ e (B) $\mathrm{OAE} 30^{\circ}$. HIS- cateter da região do feixe de His; SC- cateter do seio coronário; ABL- cateter de ablação; H1-2- par de eletrodos distal do cateter Halo na região póstero-lateral do $\mathrm{AD}$; H19-20- par de eletrodos proximal do cateter Halo na região superior septal do $\mathrm{AD}$. (fig. 3B) quando ocorriam duas frentes de ativação, sendo a colisão das frentes de ativação ao nível de AD médio (H78), demonstrando haver dificuldade de condução pelo istmo parcialmente bloqueado; c) bloqueio completo (fig. 3C) ocorre apenas uma frente de ativação em sentido anti-horário sendo o $\mathrm{AD}$ baixo lateral (H 1-2) a última região a ser ativada pelo estímulo gerado no SCP.

Durante estimulação da parede póstero-lateral do átrio direito $(600 \mathrm{~ms})$, considerou-se a ativação basal do $\mathrm{AD}$ na ausência de bloqueio. Duas ondas de ativação (septal e lateral) são observadas, partindo da parede póstero-lateral do $\mathrm{AD}(\mathrm{H} 1-2)$ e colidindo na parede lateral alta, demonstrando ausência de bloqueio anti-horário da condução na região inferior do $\mathrm{AD}$ (fig. 4A).

Foi considerado bloqueio anti-horário quando apenas uma onda de ativação ascendente percorria a parede lateral e a onda de ativação descendente era bloqueada ao nível do istmo, de maneira que o SCP era ativado após o $\mathrm{AD}$ alto e a região do His (fig. 4B).

Critérios de sucesso - Foi considerado sucesso a não indução da arritmia pós-ablação, concomitante com bloqueio completo, através da análise da ativação do impulso ao redor do anel tricuspídeo, com a verificação da concordância de ativação de todos os pares de eletrogramas analisados, indicando uma única frente de propagação do impulso.

Análise estatística - A duração dos intervalos SCP/ H1-2, pré e pós ablação, dos pacientes com bloqueio completo do istmo, foi comparada com aqueles com bloqueio incompleto ou ausência de bloqueio através da utilização da média e desvio padrão e teste não paramétrico de KruskalWallis, complementado pelo teste de comparações múltiplas de Newman Keuls. Foram comparadas as recorrências de FLA entre o grupo de bloqueio completo e o grupo de bloqueio incompleto/ausência de bloqueio utilizando-se o método de Mann-Whitney, sendo considerado um $\mathrm{p}<0,05$ estatisticamente significante.

\section{Resultados}

A ablação foi inicialmente realizada em ritmo sinusal, durante estimulação do SCP para identificação de bloqueio de condução. Em nove procedimentos houve também aplicação de RF durante FLA espontâneo. Nos pacientes que iniciaram o exame em FLA, após as primeiras aplicações de RF notou-se desaparecimento do FLA. No entanto, tais pacientes apresentavam condução intracavitária bidirecional, denotando total ausência de bloqueio do istmo. Dois pacientes com bloqueio incompleto foram submetidos à nova ablação por apresentarem recorrência do FLA.

Em sete procedimentos atingiram-se critérios parabloqueio completo, em quatro foi obtido bloqueio incompleto e em cinco não se obteve bloqueio. Naqueles onde se atingiu o bloqueio completo, em cinco, o local de sucesso foi o istmo posterior (AT-VCI) e, em dois, o istmo septal (tab. II). 


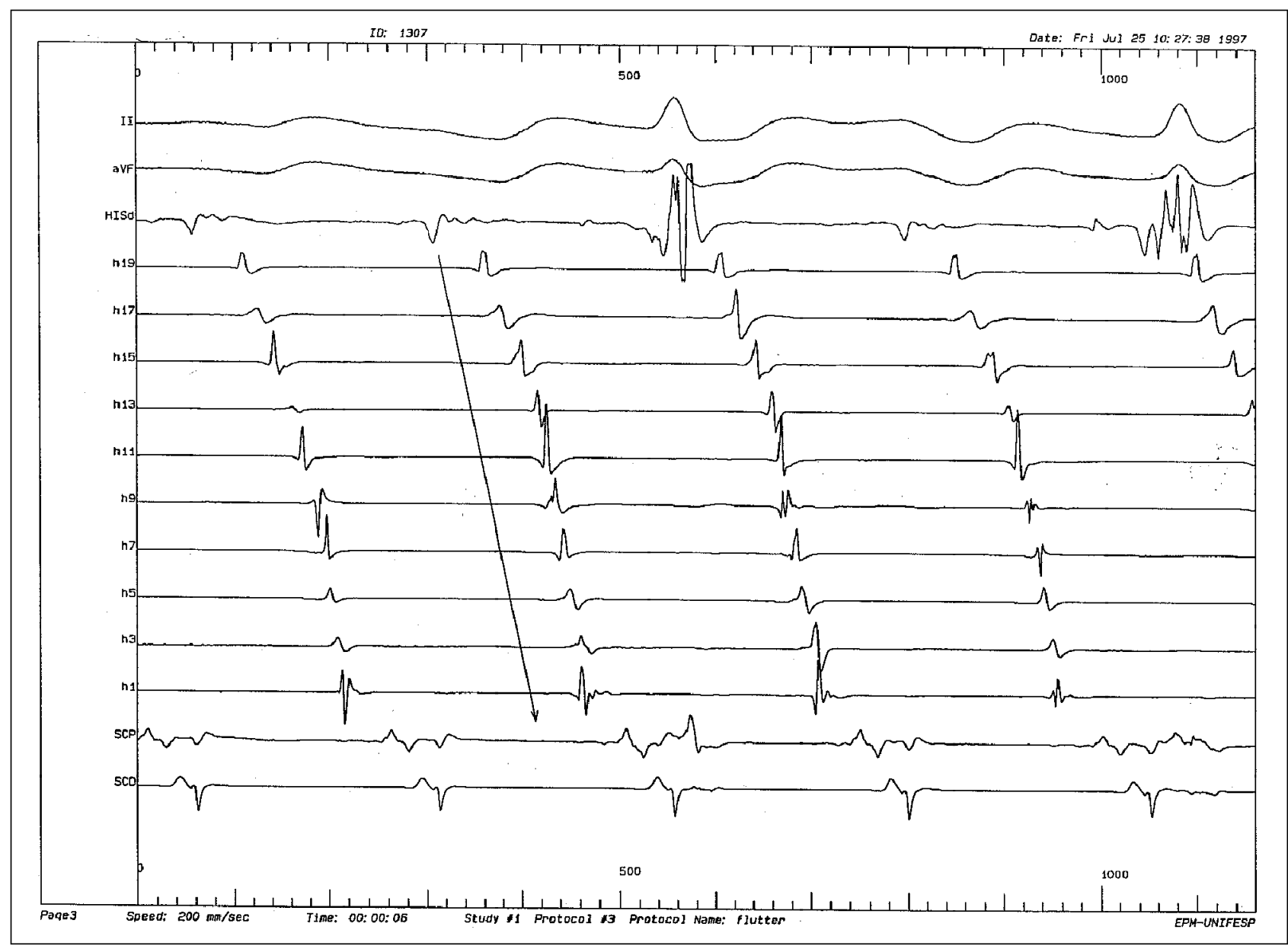

Fig. 2 - Registro intracavitário mostrando a seqüência de ativação atrial durante um flutter atrial comum. Note-se o sentido de ativação anti-horário do impulso atrial. h19- eletrodo na posição ântero-septal; h9- eletrodo em região lateral do átrio direito; h1- eletrodo em região póstero-lateral.

A ablação foi realizada durante estimulação do SCP a $600 \mathrm{~ms}$, observando-se o sentido de ativação do impulso ao redor do anel tricuspídeo. Observou-se que, à medida que procedíamos com as aplicações de radiofreqüência (RF), partindo-se de uma situação basal (fig. 3A), havia um progressivo retardo da condução ao nível do istmo, acompanhado de uma localização progressivamente mais inferior do sítio de colisão das ondas de ativação (fig. 3B), até a ocorrência de uma única frente de onda, anti-horária, pela parede lateral do $\mathrm{AD}$ (fig. $3 \mathrm{C}$ ), resultando em um prolongamento na duração do intervalo SCP/H1-2, sendo o $\Delta \mathrm{SCP} / \mathrm{H} 1-2$ (diferença entre os intervalos $\mathrm{SCP} / \mathrm{H} 1-2$ pré e pós-ablação) de $74,0 \pm 26,0 \mathrm{~ms}$, significantemente maior $(\mathrm{P}<0,001)$ que os pacientes com bloqueio incompleto $(30,5 \pm 7,5)$ ou ausência de bloqueio $(3,6 \pm 8,6)$.

Nos casos de bloqueio incompleto não se notou a presença de bloqueio unidirecional. Apenas um paciente com bloqueio completo apresentou bloqueio unidirecional horário, previamente à ablação.

Após o bloqueio completo do istmo, leves movimentos laterais do cateter de ablação (CA), durante estimulação constante do SCP, permitiram a verificação de um prolongamento importante do intervalo espícula-CA (fig. 5). Esta técnica demonstrou a presença de bloqueio ao nível do istmo.
Nos pacientes com bloqueio completo, foi verificada a presença de duplos potenciais (potenciais elétricos separados por intervalo isoelétrico, denotando bloqueio de condução) ao longo de toda a linha de ablação.

No seguimento clínico, todos os pacientes com recorrência de FLA comum apresentavam bloqueio incompleto ou ausência de bloqueio, sendo agendados para uma segunda ablação. Um deles foi submetido a novo procedimento, conseguindo-se bloqueio completo (procedimentos $\mathrm{n}^{\circ} 15$ e 16, respectivamente). A recorrência de FLA incomum (horário) foi observada em apenas um paciente com bloqueio incompleto. Esse paciente foi submetido à nova ablação, obtendo-se bloqueio completo (procedimentos $n^{\circ}$ 3 e 4 , respectivamente). Não houve recorrência naqueles indivíduos onde se conseguiu bloqueio completo.

Não foram verificadas complicações em nossa casuística. Episódios de dor torácica leve ocorreram durante a aplicação de radiofrequiência próxima ao óstio do seio coronário ou veia cava inferior.

\section{Discussão}

Os istmos septal e posterior são considerados atualmente os sítios anatômicos de predileção para realização da 


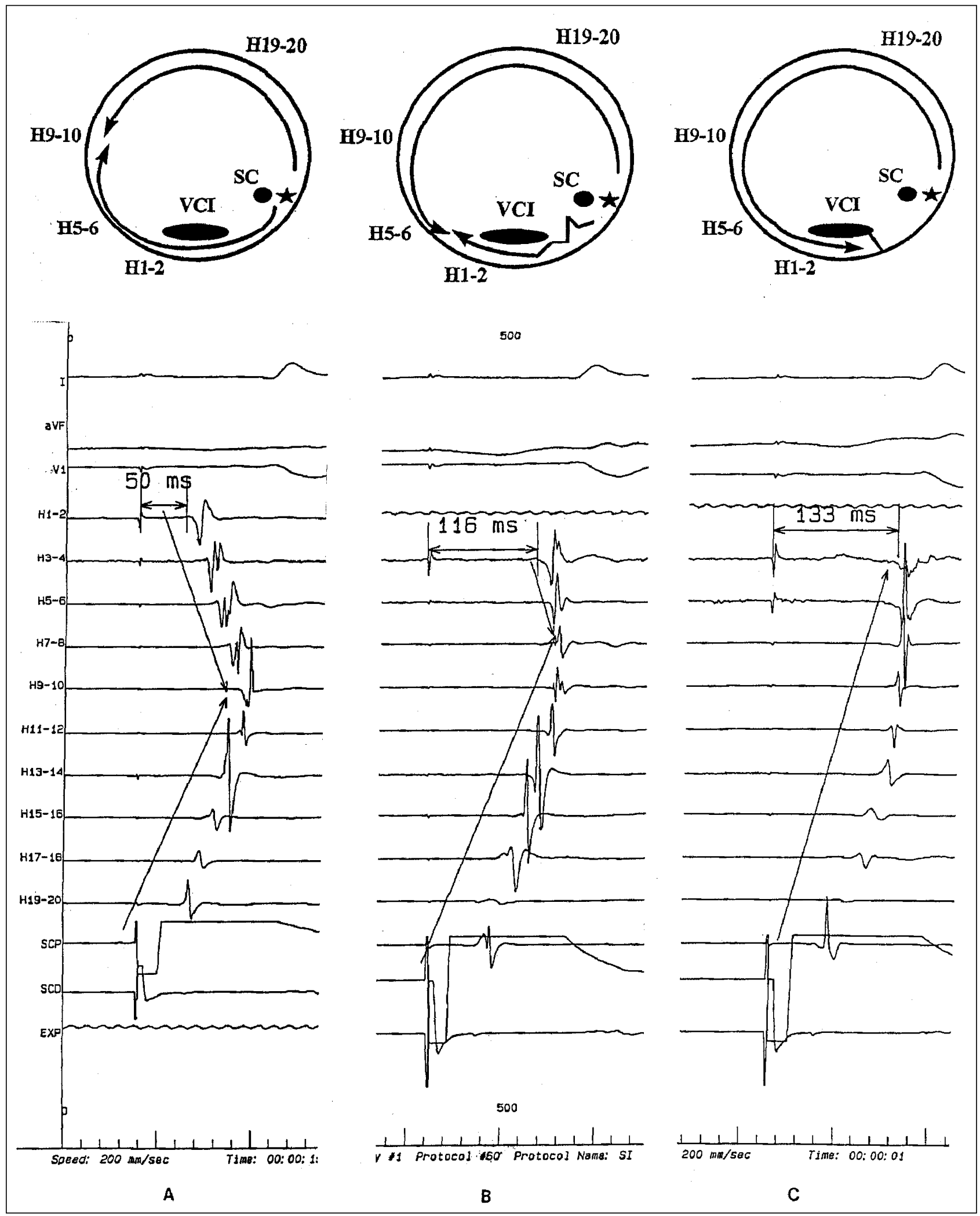

Fig. 3 - Sequiência de registros da ativação do AD durante a ablação do istmo posterior até a obtenção de um bloqueio bidirecional completo. A) ausência de bloqueio; B) bloqueio incompleto; C) bloqueio completo. VCI- veia cava inferior; $\star$ - estimulação do eletrodo posicionado na região proximal do seio coronário (SC).

linha de bloqueio durante a ablação do $\mathrm{FLA}^{7,9}$.

Apesar dos resultados imediatos satisfatórios das séries iniciais de ablação do FLA, os que utilizavam como marcador de sucesso apenas a não indutibilidade do FLA, altas taxas de recorrência dessa arritmia foram verificadas durante o seguimento clínico dos pacientes, variando de 7 a $44 \%{ }^{12,13}$.

Novos critérios eletrofisiológicos vieram se unir aos 


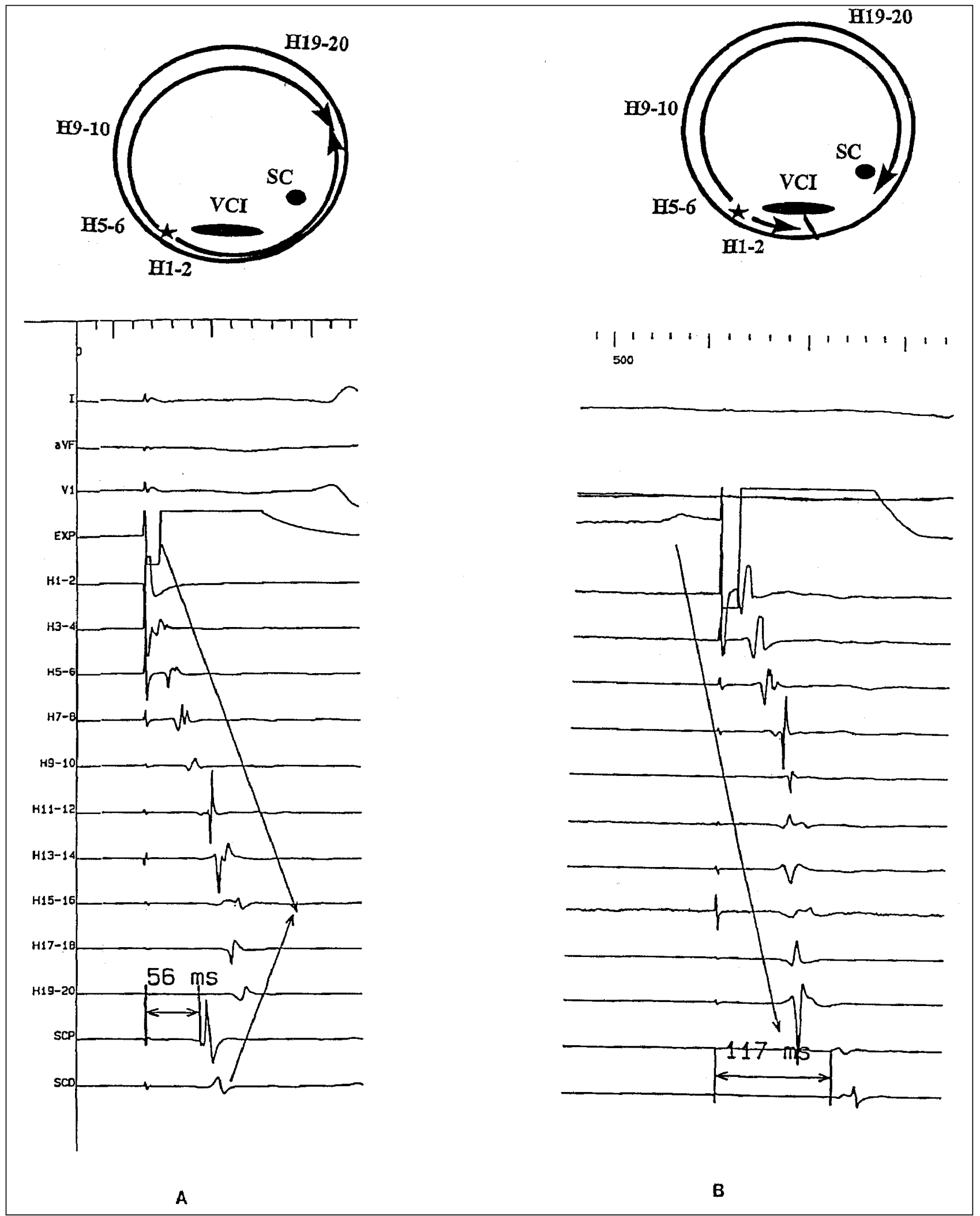

Fig. 4 - A) Estimulação da região póstero-lateral do átrio direito (H1-2) pré-ablação. O ponto de convergência das ondas de ativação se encontra em H19-20; B) estimulação da mesma região pós-ablação do istmo, notando-se um único sentido de ativação do impulso (horário) e conseqüente aumento do intervalo H1-2/SCP. VCI- veia cava inferior; SC- seio coronário.

conceitos já estabelecidos para o sucesso da ablação do FLA. Estudos recentes mostram que a realização de bloqueio bidirecional completo ao nível dos istmos posterior e/ ou septal é um excelente marcador de sucesso tardio, não apresentando recidiva de FLA tipo I num tempo de seguimento de um ano ${ }^{15,17}$. Este conceito, no entanto, necessita 


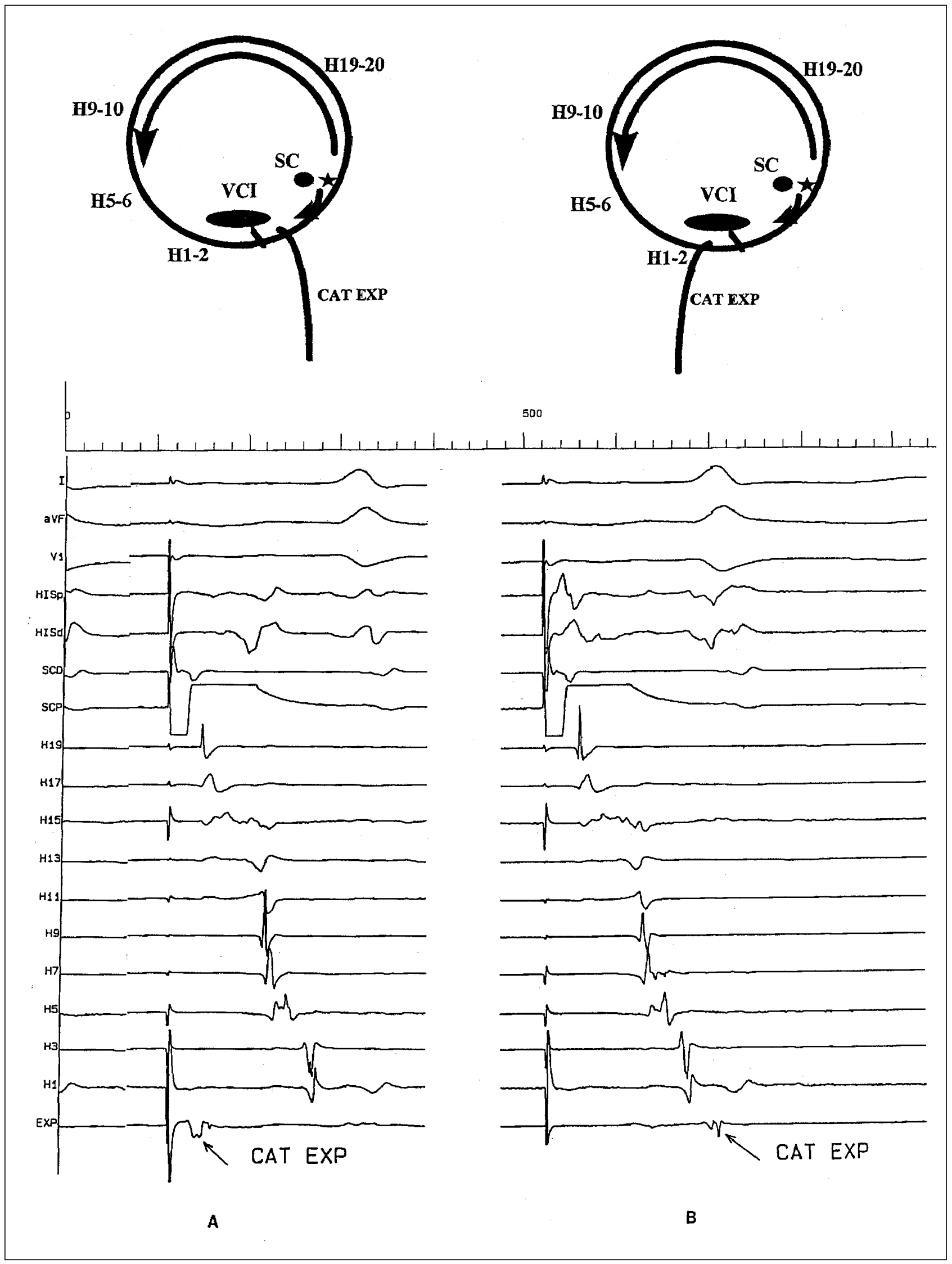

Fig. 5 - A) Estimulação do SCP, pós-ablação, com o cateter explorador (CAT EXP) posicionado medialmente à linha de bloqueio, notando-se ativação precoce do cateter explorador próxima ao sítio de estimulação; B) estimulação do SCP, com o cateter explorador posicionado lateralmente à linha de bloqueio, verificando-se sua ativação distante do sítio de estimulação. Notar que, na seqüencia de ativação atrial, esse cateter é o último a ser ativado, demonstrando um provável bloqueio completo. VCI- veia cava inferior. 


\begin{tabular}{|c|c|c|c|c|c|c|}
\hline \multicolumn{7}{|c|}{$\begin{array}{c}\text { Tabela II - Características eletrofisiológicas dos pacientes } \\
\text { submetidos à ablação de flutter atrial }\end{array}$} \\
\hline $\mathrm{N}$ & BLOQ. & $\Delta^{*}$ & NEC pré & NEC pós & ISTMO & REC.FLA \\
\hline 1 & $\mathrm{C}$ & 75 & $5 / 10$ & $10 / 10$ & POST & - \\
\hline 2 & $\mathrm{C}$ & 125 & $7 / 10$ & $10 / 10$ & SEP & - \\
\hline $3^{+}$ & I & 30 & $5 / 10$ & $8 / 10$ & SEP & Incomum \\
\hline $4^{+}$ & $\mathrm{C}$ & 55 & $7 / 10$ & $10 / 10$ & SEP & - \\
\hline 5 & $\mathrm{C}$ & 77 & $6 / 10$ & $10 / 10$ & POST & - \\
\hline 6 & $\mathrm{C}$ & 45 & $7 / 10$ & $10 / 10$ & POST & - \\
\hline 7 & $\mathrm{C}$ & 83 & $6 / 10$ & $10 / 10$ & POST & - \\
\hline 8 & A & 0 & $5 / 10$ & $5 / 10$ & POST & Comum \\
\hline 9 & A & 0 & $7 / 10$ & $7 / 10$ & POST & Comum \\
\hline 10 & I & 37 & $5 / 10$ & $7 / 10$ & SEP & Comum \\
\hline 11 & I & 35 & $6 / 10$ & $8 / 10$ & POST & - \\
\hline 12 & A & 18 & $6 / 10$ & $6 / 10$ & POST & Comum \\
\hline 13 & A & 0 & $6 / 10$ & $6 / 10$ & SEP/ POST & Comum \\
\hline 14 & A & 0 & $6 / 10$ & $6 / 10$ & SEP/ POST & Comum \\
\hline $15^{\#}$ & I & 20 & $6 / 10$ & $7 / 10$ & POST & Comum \\
\hline $16^{\#}$ & $\mathrm{C}$ & 61 & $6 / 10$ & $10 / 10$ & POST & - \\
\hline \multicolumn{7}{|c|}{$\begin{array}{l}\text { N- número do procedimento; BLOQ- bloqueio; C- completo; I- incompleto; } \\
\text { A- ausente; } \Delta \text { - diferença entre os intervalos SCP/H1-2 pré e pós ablação } \\
(\mathrm{ms}) ;\left(^{*}\right) \text { p }<0,05 \text {; NEC- }{ }^{\circ} \text { de pares de eletrogramas concordantes com o } \\
\text { mesmo sentido de ativação (pré e pós ablação); ISTMO- istmo abordado; } \\
\text { SEP- septal; POST- posterior; REC FLA- recorrência de flutter atrial; }\left(^{+}\right) \text {- } \\
\text { mesmo paciente; \# - mesmo paciente. Obs: embora o paciente }{ }^{\circ} 12 \text { tenha } \\
\text { apresentado um } \Delta \text { SCP/H1-2 de } 18 \mathrm{ms,} \mathrm{a} \mathrm{seqüência} \mathrm{de} \mathrm{ativação} \mathrm{dos} \mathrm{eletrodos} \\
\text { não apresentou qualquer modificação pós-ablação, sendo considerada ape- } \\
\text { nas uma variação decorrente da movimentação do cateter duodecapolar. }\end{array}$} \\
\hline
\end{tabular}

de um cuidadoso e detalhado mapeamento da região posterior do $\mathrm{AD}$, sendo o cateter duodecapolar (Halo) indispensável para essa tarefa.

A estimulação do SCP, em RS, durante a aplicação de $\mathrm{RF}$, combinado ao mapeamento detalhado da parede lateral e da região posterior do $\mathrm{AD}$ foi possível em todos os nossos pacientes, permitindo a visibilização do efeito da RF no istmo, demonstrando uma completa mudança no padrão de ativação atrial direita (crânio-caudal ou caudocranial), indicando, desta maneira a presença de bloqueio do istmo.

A simples criação de um bloqueio intra-atrial na região inferior do $\mathrm{AD}$, no entanto, não é suficiente para garantir a ausência de condução através dessa região, uma vez que pode haver apenas retardo da condução. No bloqueio incompleto, quando estimulamos o SCP, a porção lateral do sítio de ablação no istmo (H1-2) é despolarizada antes do AD alto (H9-10), revelando condução residual na região do istmo. Este tipo de ativação excêntrica (do septo à região lateral do $\mathrm{AD}$ ), durante a estimulação do SCP pode mimetizar o padrão de ativação encontrado no bloqueio completo, através de um retardo de condução no AD baixo. Somente um mapeamento detalhado da porção lateral ao istmo permite a distinção entre os dois tipos de

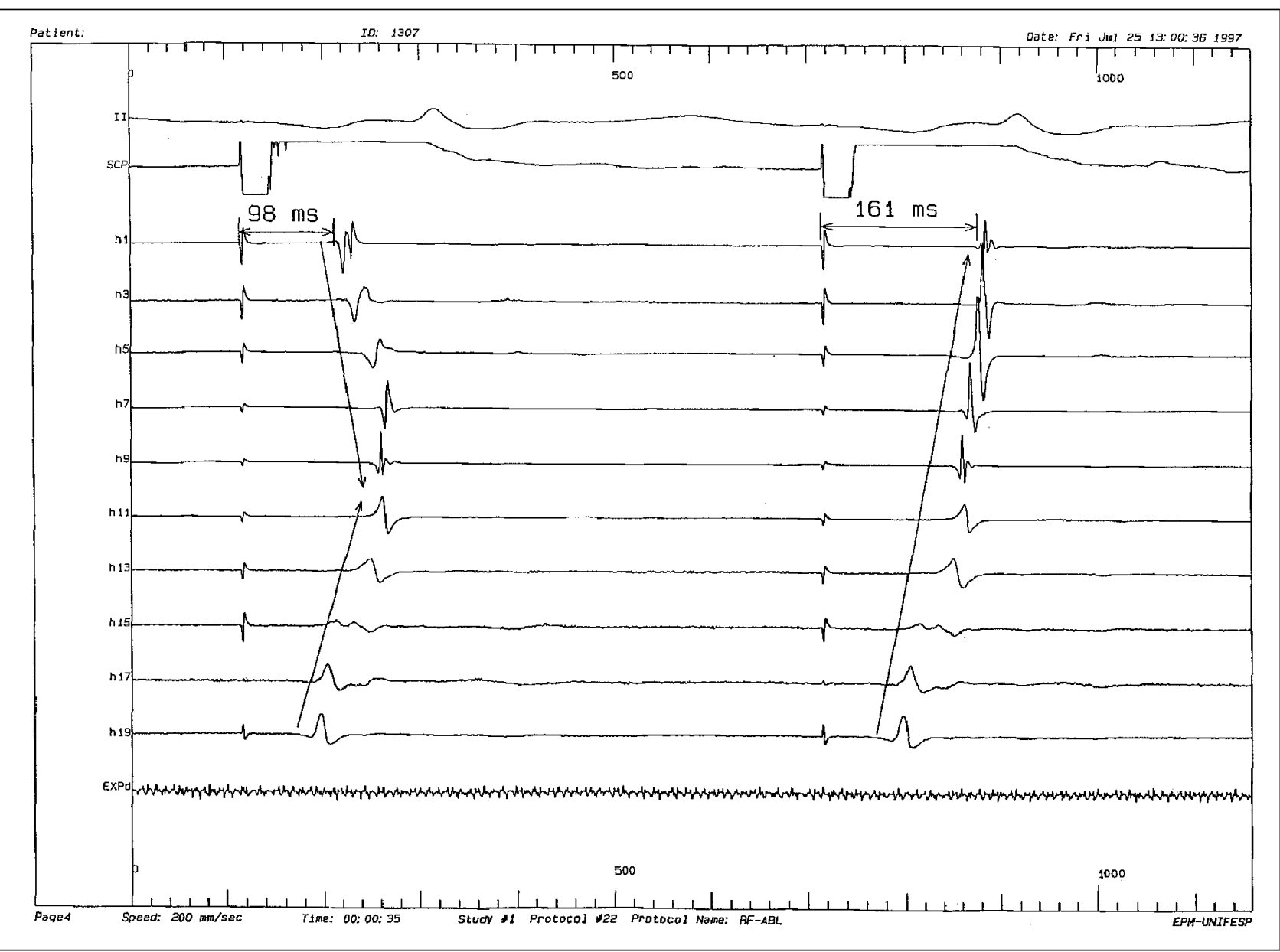

Fig. 6 - Demonstração de bloqueio horário completo durante ablação do istmo posterior, sob estimulação do SCP. Neste caso, podemos notar a brusca mudança no sentido de ativação do impulso, e conseqüente aumento do intervalo SCP/H1-2 (de 98ms para 161ms), durante a aplicação de RF. 


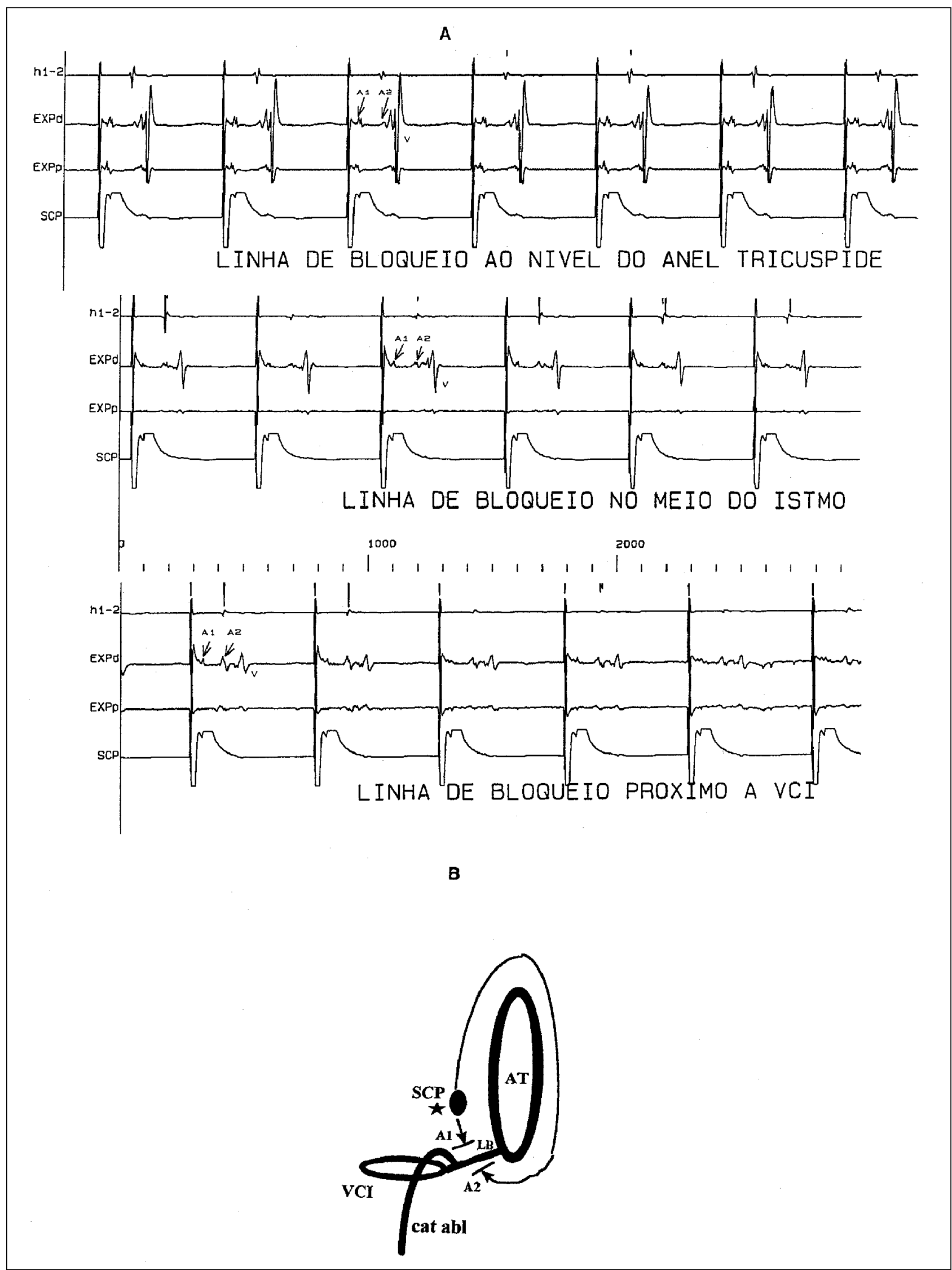

Fig. 7 - A) Eletrograma intracavitário demonstrando a presença de duplos potenciais, sob estimulação do SCP, ao longo do istmo posterior, após ablação linear. Tal registro foi caracterizado durante o recuo do cateter explorador desde o anel tricuspídeo (notar eletrograma ventricular amplo), até a veia cava inferior (quase ausência de eletrograma ventricular); v- eletrograma ventricular; B) figura ilustrativa mostrando a linha de bloqueio pós-ablação e a presença de duplos potenciais; A1- bloqueio anterior da condução; A2bloqueio posterior da condução. VCI- veia cava inferior; AT- anel tricuspídeo; SCP- seio coronário proximal; LB- linha de bloqueio; Cat abl- cateter de ablação. 
bloqueio. Na nossa experiência, a utilização de cateteres multipolares especiais, como o utilizado em nosso estudo, é fundamental para o registro das informações eletrofisiológicas que definem os bloqueios dos istmos posterior ou septal.

A duração do intervalo SCP/H1-2 não foi discriminante para diferenciar o bloqueio completo do incompleto, porém, o $\Delta \mathrm{SCP} / \mathrm{H} 1-2$ foi significantemente maior nos indivíduos com bloqueio completo. As variações desse intervalo nos pacientes com bloqueio incompleto estão na dependência direta do tempo de condução através do istmo parcialmente danificado pela ablação. Já nos pacientes com bloqueio completo, as variações individuais desse intervalo podem ser explicadas pela sua dependência nas propriedades de condução intra-atrial e também na dimensão do $\mathrm{AD}$, o que explicaria por que no procedimento $\mathrm{n}^{\circ} 6$, apesar de encontrarmos um $\Delta \mathrm{SCP} / \mathrm{H} 1-2$ de apenas $45 \mathrm{~ms}$, foi encontrado bloqueio completo, levando-nos a associar tal intervalo às reduzidas dimensões do átrio desse paciente. Nesses casos, a presença de concordância absoluta da ativação, através da visibilização da seqüência de ativação dos eletrogramas, foi determinante para o diagnóstico de bloqueio completo. A duração desse intervalo também depende do posicionamento do eletrodo distal do cateter Halo (H1-2) na região inferior do $\mathrm{AD}$ e do eletrodo proximal do cateter do seio coronário em relação ao óstio.

A variação dos valores correspondentes ao intervalo SCP/H1-2 não nos permite estabelecer um valor crítico que possa diferenciar bloqueio completo de incompleto. Esta discriminação só é possível pelo cuidadoso mapeamento da seqüência de ativação de cada lado da linha de bloqueio, havendo sempre a necessidade de perseguir uma completa unificação do sentido de ativação em todos os eletrogramas atriais registrados no cateter duodecapolar. Na nossa casuística, o $\Delta \mathrm{SCP} / \mathrm{H} 1-2 \mathrm{de} 45 \mathrm{~ms}$ foi o menor valor encontrado nos casos de bloqueio completo, enquanto que naqueles indivíduos com bloqueio incompleto, esse valor não ultrapassou os $37 \mathrm{~ms}$.

A realização da ablação do istmo, estimulando-se o SCP em RS, deve ser sempre considerada, especialmente nos pacientes que não toleram o FLA e naqueles suscetíveis à fibrilação atrial. Esta técnica possibilita ainda a verificação, em tempo real, do bloqueio completo, através da observação de um "salto" no intervalo SCP/H1-2, bem como da brusca mudança no sentido de ativação dos eletrodos bipolares, durante a aplicação de RF (fig. 6).

Segundo Poty e col, embora o deslocamento do cateter de ablação, da região medial à lateral da linha de bloqueio, demonstre a presença de bloqueio no istmo, ele não discrimina o bloqueio completo do incompleto, uma vez que não se pode visibilizar quão distante dessa linha esse cateter se localiza. Possivelmente, os movimentos laterais utilizados nessa estratégia, podem ser intensos a ponto de o distanciarem da linha de bloqueio e, dessa forma, não identificar uma possível condução lenta através do istmo ${ }^{15}$.
Os “duplos potenciais" são potenciais elétricos separados por um intervalo isoelétrico, consistente com bloqueio de condução. Esses potenciais foram registrados ao longo da válvula de Eustáquio (entre a margem do óstio do seio coronário e a veia cava inferior) e outras estruturas anatômicas que servissem de barreira natural à condução durante o FLA. Sua presença denota duas ondas de ativação se encontrando de cada lado da barreira anatômica, anteriormente e posteriormente ao estímulo ${ }^{9,19}$. A verificação desses potenciais se dá durante o FLA ou sob estimulação com um ciclo de freqüência mais rápida do que o ritmo sinusal, não sendo verificada em ritmo sinusal, possivelmente por uma ativação simultânea de ambos os lados da barreira anatômica. Dessa forma, pudemos observar em alguns pacientes a presença de uma linha de bloqueio "artificial", criada pela aplicação de energia de RF ao longo do istmo abordado. Após a aplicação da RF, mapeamos todo o istmo à procura de uma possível falha da linha (ausência de duplos potenciais ou presença de potenciais fracionados ou únicos), alvo certo para uma nova aplicação de $\mathrm{RF}^{20}$. A constatação de duplos potenciais ao longo de toda região de aplicação de RF confirma a presença de uma linha de bloqueio sem falhas (fig. 7).

Bloqueios de condução bidirecional podem ser considerados completos ou aparentemente completos (com maior alentecimento) podendo ser ainda transitórios ou permanentes ${ }^{18}$.

Dessa forma, a estimulação do SCP, em ritmo sinusal, acompanhado de um detalhado mapeamento da parede lateral e região inferior do $\mathrm{AD}$, mostrou ser uma estratégia eficaz na discriminação de bloqueio completo de incompleto, uma vez que permite visibilizar toda a ativação na região lateral à linha de bloqueio, desmascarando, assim, possíveis "pseudo" bloqueios bidirecionais.

Em nossa casuística os, sete pacientes que obtiveram bloqueio bidirecional completo não mais apresentaram recidiva de FLA num seguimento médio de um ano, independentemente do istmo abordado. Entretanto, todos os quatro pacientes com ausência de bloqueio e três dos quatro pacientes com bloqueio incompleto apresentaram recorrência do FLA nos dois primeiros meses, não havendo diferença entre os tempos médios de recorrência entre estes dois grupos. No entanto, comparando-se o grupo bloqueio completo com o grupo bloqueio incompleto/ausência de bloqueio, pudemos notar uma diferença significante $(\mathrm{p}<0,001)$. As variáveis idade, sexo e fração de ejeção medida pelo ecocardiograma não apresentaram qualquer influência nos resultados da ablação.

A utilização desses novos marcadores eletrofisiológicos mostrou-se ser de grande utilidade para validação de bloqueio completo do istmo atrial (posterior ou septal), na ablação do FLA tipo I. A realização desse bloqueio está relacionada a um sucesso a longo prazo, devendo ser perseguida sempre que possível. O emprego de cateteres multipolares especiais é fundamental para definir com precisão os tipos de bloqueio, garantindo um excelente resultado da ablação. 


\section{Referências}

1. Lewis T, Feil HS, Stroud WD - Observations upon flutter and fibrillation, II: the nature of auricular flutter. Heart 1920; 7: 191-245.

2. Rosenblueth A, Garcia-Ramos J - Studies on flutter and fibrillation, II: the influence of artificial obstacles on experimental auricular flutter. Am Heart J 1947; 33: 677-84.

3. Waldo AL, McLean WAH, Karp RB, Kouchoukos NT - Entrainment and interruption of atrial flutter with pacing: studies in man following open heart surgery. Circulation 1977; 56: 737-45.

4. Wells JL, McLean WAH, James TN, Waldo AL - Characterization of atrial flutter: studies in man after open heart surgery using fixed atrial electrodes. Circulation 1979; 60: 665-73.

5. Boineau JP, Schuessler RB, Mooney CR et al - Natural and evoked atrial flutter due to circus movement in dogs. Am J Cardiol 1980; 45: 1167-81.

6. Cox JL, Schuessler RB, Boineau JP - The surgical treatment of atrial fibrillation, I: summary of the current concepts of the mechanisms of atrial flutter and atrial fibrillation. J Thorac Cardiovasc Surg 1991; 101: 402-5.

7. Cosio FG, Lopez-Gil M, Goicolea A, Arribas F, Barroso JL - Radiofrequency ablation of the inferior vena cava-tricuspid valve isthmus in common atrial flutter. Am J Cardiol 1993; 71: 705-9.

8. Lesh MD, Van Hare GF, Epstein LM et al - Radiofrequency catheter ablation of atrial arrhytmias: results and mechanisms. Circulation 1994; 89: 1074-89.

9. Nakagawa H, Lazzara R, Khastgir T et al - Role of the tricuspid annulus and the eustachian valve/ridge on atrial flutter. Relevance to catheter ablation of the septal isthmus and a new technique for rapid identification of ablation sucess. Circulation 1996; 94: 407-24.

10. Calkins H, Leon AR, Deam AG et al - Catheter ablation of atrial flutter using radiofrequency energy. Am J Cardiol 1994; 73: 353-6.

11. Feld GK, Fleck RP, Chen PS et al - Radiofrequency catheter ablation for the treatment of humam type 1 flutter. Identification of a critical zone in the reentrant circuit by endocardial mapping techniques. Circulation 1992; 86: 1233-40.

12. Interian A, Cox M, Jimenez RA et al - A shared pathway in atrioventricular nodal reentrant tachycardia and atrial flutter: implications for pathophysiology and therapy. Am J Cardiol 1993; 71: 297-303.

13. Nakagawa H, McClelland J, Beckman K et al - Radiofrequency catheter ablation of common type atrial flutter. PACE 1993; 16: II-85.

14. Poty H, Saoudi N, Aziz AA, Nair M, Letac B - Radiofrequency catheter ablation of type 1 atrial flutter. Prediction of late success by electrophysiological criteria. Circulation 1995; 92: 1389-92.

15. Poty H, Saoudi N, Nair M, Anselme F, Letac B. Radioquency catheter ablation of atrial flutter. Further insights into the various types of isthmus block: application to ablation during sinus rhytm. Circulation 1996; 94: 3204-13.

16. Waldo AL - Atrial flutter: Entrainment characteristics. J Cardiovasc Eletrophysiol 1997; 8: 337-52.

17. Poty H, Saoudi N, Abdel Aziz et al - Radiofrequency catheter ablation of type 1 flutter: prediction of late sucess by electrophysiological criteria. Circulation 1995; 92: 1389-92.

18. Barold SS, Shah D, Jais P et al - Nomenclature and characterization of transisthmus conduction after ablation of typical atrial flutter. PACE 1997; 20: 1751-3.

19. Olgin JE, Kalman JM, Fitzpatrick AP et al - Role of right atrial endocardial structures as barriers to conduction during human type I atrial flutter: activation and entrainment mapping guided by intracardiac echocardiography. Circulation 1995; 92: 1839-48.

20. Shah DC, Haissaguerre M, Jais P et al - Simplified electrophysiologically directed catheter ablation of recorrent common atrial flutter. Circulation 1997; 96: 2505-8. 